\title{
Zurich 1940
}

\section{François Sigrist}

François Sigrist a étudié les mathématiques à l'EPFZ, où il a obtenu le doctorat en 1967 sous la direction de Beno Eckmann. Il a enseigné à UBC Vancouver avant d'être nommé à l'Université de Neuchâtel, où il a enseigné jusqu'en 2006. Il a consacré sa recherche à la topologie algébrique (H-espaces, K-théorie, homotopie) pendant une vingtaine d'années, puis aux formes quadratiques réelles, aux empilements de sphères et aux codes correcteurs d'erreurs.

\section{Introduction}

Nous sommes à Zurich, en 1940, à l'Ecole polytechnique fédérale (EPF, en allemand ETH, devenue en 1971 EPFZ-ETHZ lors de la création de l'EPFL à Lausanne). Depuis sa création en 1855 , l'institution est connue sous le nom de «Poly».

Les trois protagonistes de cette histoire sont Heinz Hopf, Eduard Stiefel, et Beno Eckmann. Hopf est professeur au Poly. Il est au sommet de son art, et au firmament des mathématiques. Stiefel est un de ses collaborateurs, il a publié une remarquable thèse, sous sa direction, en 1935. Eckmann termine sa thèse, également dirigée par Hopf, et tout aussi remarquable (il a 23 ans).

Ces trois mathématiciens ont aujourd'hui leur place parmi les grands. Leurs travaux de l'époque, et à venir, sont justement célèbres.

J'ai sélectionné pour chacun d'eux un article qui, de leur point de vue, à ce momentlà, n'avait peut-être pas l'importance qu'il a acquise plus tard. Mais dans chaque cas, on trouve une extraordinaire étincelle de clairvoyance qui force l'admiration.

Meistens wird Eulers Polyedersatz als Ursprung der algebraischen Topologie betrachtet. Als einer der Gründungstexte darf Poincarés Analysis Situs von 1895 gelten. In den 30er Jahren des 20. Jahrhunderts hat die algebraische Topologie eine ausserordentliche Entwicklungsphase durchgemacht. Einer der kreativsten Protagonisten jener Zeit war Heinz Hopf. Er hat damals mehrere Forschungsrichtungen initiiert, die sich in der Folge als äusserst fruchtbar erweisen sollten. Der Autor hat den Jahrgang seiner Geburt zum Anlass genommen, um die damalige Stimmung am Poly Zürich zu beschreiben, als man, mitten im Krieg, mathematisch zu neuen Ufern aufbrach. 
Mon choix est en bonne partie dicté par des raisons personnelles. J'ai eu la chance, au cours de mes études au Poly, de bénéficier de l'enseignement de ces trois professeurs, et j'en conserve un souvenir durable. Eckmann a été mon directeur de thèse. Je suis né en 1940.

\section{Heinz Hopf (1894-1971)}

Hopf fait paraître dans une revue locale (Bulletin de la société zurichoise des sciences naturelles) un article intitulé „Systeme symmetrischer Bilinearformen und euklidische Modelle der projektiven Räume“. Il commence par énoncer une propriété en apparence tout à fait élémentaire :

Quatre coniques dans le plan ont toujours une paire commune de points conjugués.

Tout en laissant entendre que ce résultat est bien connu, il met en bas de page, dans son style caractéristique, et avec une pointe d'humour, la note suivante (traduire serait un crime) :

„Bei dem Versuch, diese Sätze mit den üblichen Methoden der projektivenalgebraischen Geometrie zu beweisen - gewiss ist ein solcher Beweis möglich - macht, soviel ich sehe, die notwendige Realitätsbetrachtung einige Schwierigkeit.“ 1

Appelons $N(r)$ le nombre minimal nécessaire de quadriques, dans l'espace projectif réel de dimension $(r-1)$, permettant de garantir qu'il n'y ait aucune paire commune de points conjugués. Avec cette notation, la propriété s'énonce $N(3)>4$.

Hopf s'apprête alors à démontrer :

$$
\text { Pour } \quad r>2, \quad r+2 \leq N(r) \leq 2 r-1 .
$$

Voici la démonstration de $N(3)=5$ donnée par Hopf. Elle a la vertu d'être élémentaire, tout en renseignant valablement sur le cas général traité. Mais au préalable, quelques remarques sur la géométrie projective, qui a peu à peu disparu de l'enseignement, à tous les niveaux de formation, alors qu'elle joue un rôle important, en géométrie algébrique par exemple. Je prendrai l'exemple de la dimension 2, puisque c'est le contexte de l'énoncé de départ.

Le plan projectif réel $\mathbb{R P}^{2}$ est défini comme l'espace des droites par l'origine dans $\mathbb{R}^{3}$. On introduit les coordonnées homogènes pour le décrire : un point du plan projectif est un triple de nombres réels $\left[x_{1}, x_{2}, x_{3}\right]$, différent de $[0,0,0]$, avec l'identification de $\left[x_{1}, x_{2}, x_{3}\right]$ avec $\left[\lambda x_{1}, \lambda x_{2}, \lambda x_{3}\right]$. La géométrie projective plane consiste donc à adjoindre au plan euclidien $\mathbb{R}^{2}$ une droite à l'infini. Chaque droite du plan a un seul point à l'infini, ce qui rend l'espace projectif non-orientable : un «ruban» du plan, avec ses points à l'infini, est un ruban de Möbius !

Un point $p=\left(\begin{array}{c}p_{1} \\ p_{2}\end{array}\right)$ du plan euclidien correspond au point $\left[p_{1}, p_{2}, 1\right]$ dans le plan projectif. Il est commode d'utiliser les mêmes notations, j'écrirai donc $p=\left(\begin{array}{c}p_{1} \\ p_{2} \\ 1\end{array}\right)$ dans le plan projectif. Remarque : la droite à l'infini est l'ensemble des points $\left[x_{1}, x_{2}, 0\right]$.

1. Malgré mes bons souvenirs, je ne sais absolument pas démontrer ce résultat avec les méthodes traditionnelles de la géométrie projective. 
Dans le plan euclidien, une conique est un polynôme de degré 2 en $x$ et $y$. Il lui correspond dans le plan projectif un polynôme homogène de degré 2 en $x, y$ et $z$, de matrice (symétrique réelle) $3 \times 3$. En notant $A$ la matrice de la conique, $X=\left(\begin{array}{l}x \\ y \\ z\end{array}\right)$ le point du plan projectif, l'équation de la conique est donc

$$
X^{t} A X=0 .
$$

La polaire d'un point $P$ par rapport à la conique de matrice $A$ est la droite d'équation $P^{t} A X=0$. Cette dualité est l'un des outils standard de la géométrie projective, elle permet de transcrire les résultats pour les points en résultats pour les droites. Echantillon : Par 5 points, il passe une et une seule conique. Par conséquent, une conique est aussi caractérisée par 5 tangentes.

Deux points $P$ et $Q$ sont conjugués par rapport à la conique de matrice $A$ si $P$ est situé sur la polaire de $Q: P^{t} A Q=0$.

Avec ces notations, voici comment Hopf procède :

Il commence par calculer le produit de deux polynômes (en $t$ )

$$
\begin{aligned}
\left(x_{1}+y_{1} t+z_{1} t^{2}\right)\left(x_{2}\right. & \left.+y_{2} t+z_{2} t^{2}\right)=\left(x_{1} x_{2}\right)+\left(x_{1} y_{2}+x_{2} y_{1}\right) t \\
& +\left(x_{1} z_{2}+x_{2} z_{1}+y_{1} y_{2}\right) t^{2}+\left(y_{1} z_{2}+y_{2} z_{1}\right) t^{3}+\left(z_{1} z_{2}\right) t^{4} .
\end{aligned}
$$

Le produit de deux polynômes non-nuls n'étant jamais le polynôme nul, les points du plan projectif $\left[x_{1}, y_{1}, z_{1}\right]$ et $\left[x_{2}, y_{2}, z_{2}\right]$ ne peuvent pas annuler simultanément les cinq coefficients du produit. Ils ne peuvent donc pas former une paire de points conjugués pour les cinq coniques correspondantes dont les équations sont

$$
x^{2}=0, \quad 2 x y=0, \quad 2 x z+y^{2}=0, \quad 2 y z=0, \quad z^{2}=0 .
$$

Il en déduit évidemment que $N(3) \leq 5$. $^{2}$

Il suppose ensuite que $N(3)=4$. Il existe alors quatre matrices $3 \times 3, A_{1}, A_{2}, A_{3}, A_{4}$, réelles, symétriques, telles que

$$
X^{t} A_{i} Y=0 \quad(i=1,2,3,4) \Longrightarrow X=0 \text { ou } Y=0 .
$$

On exploite cette propriété en construisant une application $\phi: \mathbb{R}^{3}-\{0\} \rightarrow \mathbb{R}^{4}$ donnée par

$$
\phi(X)=\frac{\left(X^{t} A_{1} X, X^{t} A_{2} X, X^{t} A_{3} X, X^{t} A_{4} X\right)}{\sqrt{\left(X^{t} A_{1} X\right)^{2}+\left(X^{t} A_{2} X\right)^{2}+\left(X^{t} A_{3} X\right)^{2}+\left(X^{t} A_{4} X\right)^{2}}} .
$$

On constate alors avec ravissement que l'application $\phi$ est constante sur les droites par l'origine de $\mathbb{R}^{3}$, qu'elle a son image sur la sphère $\mathbb{S}^{3}$ et qu'elle n'est pas surjective sur $\mathbb{S}^{3}$ pour raison dimensionnelle ( $\phi$ est différentiable). Autrement dit, $\phi$ est une application du plan projectif $\mathbb{R P}^{2}$ dans $\mathbb{R}^{3}$. Mais à ce titre, $\phi$ est injective. En effet, $\phi(X)=\phi(Y)$ implique qu'il existe $\lambda \neq 0$ avec $\left(X^{t} A_{i} X\right)=\lambda^{2}\left(Y^{t} A_{i} Y\right), i=1,2,3$, 4. Les matrices étant symétriques, il vient $(X-\lambda Y)^{t} A_{i}(X+\lambda Y)=0, i=1,2,3$, 4. Par conséquent $X= \pm \lambda Y$, ou encore $X=Y$ dans $\mathbb{R P}^{2}$.

2. L'argument général donne $N(r) \leq 2 r-1$. De plus, en prenant des polynômes à coefficients complexes, on obtient $N(2 q) \leq 4 q-2$. 
Dans le langage forgé par Hopf, le plan projectif $\mathbb{R P}^{2}$ possède un modèle euclidien de dimension 3. Or, il est bien connu que c'est impossible, puisque le plan projectif n'est pas orientable. Pour le cas général, Hopf utilise la dualité d'Alexander et obtient $N(r) \geq$ $r+2$. C'est donc une démonstration «clandestinement cohomologique», alors que le mot «cohomologie»n'a jamais fait partie de son vocabulaire.

Le problème topologique sous-jacent est donc : Trouver la dimension $D(m)$ du plus petit espace euclidien dans lequel on puisse plonger l'espace projectif $\mathbb{R P}^{m}$. La contribution de Hopf est donc $m+2 \leq D(m) \leq 2 m$.

Le problème est resté d'actualité. Des centaines d'articles ont été écrits sur la borne inférieure. Les distinctions de cas sont innombrables, les démonstrations sont souvent très ardues. Pour la borne supérieure, je ne connais qu'un résultat général, spectaculaire, dû à Brian Steer (1970): $D(m) \leq 2 m-\alpha(m)+1$. La fonction $\alpha(m)$ compte le nombres de $1^{\prime} s$ dans l'écriture de $m$ en base deux. Sa pertinence dans le problème vient du fait que la borne inférieure conjecturale est $D(m) \geq 2 m-2 \alpha(m)$. Il y a bien des années, j'ai assisté, et un peu participé, aux efforts de Brian Steer et d'Ueli Suter pour rétablir une démonstration incorrecte qui venait de paraître de cette conjecture, sans succès. Mais il est probable qu'on puisse la confirmer aujourd'hui, simplement en compilant la masse de données à disposition. Le problème est remarquablement tenu à jour sur

www. lehigh. edu/ dmal/immtable.

\section{Eduard Stiefel (1909-1978)}

L'article dont je vais parler s'intitule „Über Richtungsfelder in den projektiven Räumen und einen Satz aus der reellen Algebra“. L'auteur est bien Stiefel, mais une note en bas de la première page mérite attention.

Il y a bien des disciplines scientifiques où les patrons signent simplement les articles des chercheurs. Ce n'est pratiquement jamais le cas en mathématiques. Malgré cela, il est tout de même rare que le patron écrive l'article du chercheur. Voici le texte intégral, qui illustre bien le climat de l'époque :

„Herr Prof. Dr. H. Hopf war so freundlich, mein ursprünglich vorliegendes Manuskript während meiner längeren Abwesenheit im Militärdienst für den Druck auszuarbeiten. Er hat bei dieser Gelegenheit einige Hilfsmittel, die ich als Spezialfälle der allgemeinen Theorie dargestellt hatte, für die projektiven Räume direkt hergeleitet. Ferner hat er einen Beweis, der bei mir noch nicht ganz präzis war, durch Benutzung eines Satzes von Wasewski in Ordnung gebracht. Ich danke ihm herzlich für seine Hilfe, ohne die das Erscheinen der Arbeit zum mindesten stark verzögert worden wäre.“

Il est fascinant de lire cet article avec le recul que donnent les années, car il montre la genèse de notions qui ont complètement envahi la topologie algébrique. Les résultats se sont «banalisés» au point d'apparaître comme exercices dans les livres sur le sujet.

Au préalable, et pour lier les problèmes envisagés ici, une petite parenthèse sur le théorème du hérisson (Igelsatz) me semble utile. Il est impossible de peigner un hérisson : il n'existe pas de champ de vecteurs tangents à la sphère $\mathbb{S}^{2}$ et non-nuls en tout point de $\mathbb{S}^{2}$. Ce résultat 
de Poincaré (démontré pour toute sphère de dimension paire) est le point de départ de toute la topologie algébrique du siècle précédent.

Hopf, après avoir démontré le théorème du hérisson, aimait en présenter la conséquence suivante, qui est un cas particulier des résultats de Stiefel que nous verrons ci-après :

S'il existe deux matrices réelles $(n \times n)$ telles que toutes leurs combinaisons linéaires non-triviales aient le rang $n$, alors $n$ est pair.

Preuve. On peut supposer que l'une des deux matrices est la matrice-unité. Appelons $A$ l'autre. Pour tout vecteur-unité $v$ de $\mathbb{R}^{n}$, les vecteurs $v$ et $A v$ sont linéairement indépendants. En projetant $A v$ sur le plant tangent à la sphère-unité, on obtient un champ de vecteurs tangents non-nuls.

Eckmann préférait, par principe, énoncer les résultats sous forme positive. Sa version était donc :

S'il existe $2 m+1$ fonctions réelles de $2 m+1$ variables

telles que

$$
f_{1}\left(x_{1}, \ldots, x_{2 m+1}\right), \ldots, f_{2 m+1}\left(x_{1}, \ldots, x_{2 m+1}\right)
$$

$$
x_{1} f_{1}+x_{2} f_{2}+\ldots+x_{2 m+1} f_{2 m+1} \equiv 0
$$

alors les fonctions $\left(f_{1}, \ldots, f_{2 m+1}\right)$ ont un zéro commun autre que l'origine.

Preuve. A contrario, le vecteur $\left(f_{1}, f_{2}, \ldots, f_{2 m+1}\right)$, orthogonal au vecteur-unité $\left(x_{1}, x_{2}\right.$, $\left.\ldots, x_{2 m+1}\right)$, ne s'annule pas en dehors de l'origine, il constitue donc un champ de vecteurs tangents à la sphère de dimension $2 m$.

Voici maintenant un des résultats de l'article de Stiefel, dans sa version originale :

Théorème. S'il existe s matrices $(r \times n)$ telles que toute combinaison linéaire non-triviale ait le rang $r$, il est nécessaire que les coefficients binomiaux $\left(\begin{array}{l}n \\ j\end{array}\right)$ soient pairs pour $n-r<$ $j<s$.

Remarque : La condition donnée est équivalente à

$$
(x+y)^{n}=0 \quad \text { dans } \quad \mathbb{F}_{2}[x, y] /\left(x^{r}, y^{s}\right) .
$$

Le cas $r=n$ fournit $s-1$ champs de vecteurs tangents à la sphère $\mathbb{S}^{n-1}$, linéairement indépendants en tout point. Par exemple, $s=2$ demande que $\left(\begin{array}{l}n \\ 1\end{array}\right)$ soit pair, donc que $n$ soit pair, c'est la conséquence décrite ci-dessus du théorème du hérisson. C'est pourtant le cas $s=r=n$ qui est le plus intéressant. Il existe alors $n-1$ champs de vecteurs tangents à la sphère $\mathbb{S}^{n-1}$, linéairement indépendants en chaque point. On dit alors que la sphère $\mathbb{S}^{n-1}$ est parallélisable : on peut employer les mêmes coordonnées dans tous les espaces tangents. Il est donc nécessaire que $\left(\begin{array}{c}n \\ j\end{array}\right)$ soit pair pour $0<j<n$, ou encore que $n$ soit une puissance de 2. Ce résultat était probablement déjà déductible de la thèse de Stiefel en 1935, il est d'une importance historique considérable.

Seules les sphères $\mathbb{S}^{1}, \mathbb{S}^{3}$, et $\mathbb{S}^{7}$ sont parallélisables, et ceci grâce à leur structure multiplicative (nombres complexes, quaternions, et octaves de Cayley). Il suffit en effet de déplacer l'espace tangent par multiplication pour obtenir un parallélisme global. Mais il a fallu attendre 1958 (Milnor et Kervaire, séparément) pour montrer que ce sont les seules sphères parallélisables. 


\section{Beno Eckmann (1917-2008)}

Le cas particulier du problème posé par Stiefel : Trouver $s$ matrices inversibles $n \times n$ telles que toute combinaison linéaire soit inversible, avait été résolu séparément par A. Hurwitz et par J. Radon en 1923, dans le cas où les matrices sont orthogonales. En voici le résultat.

Théorème (Hurwitz-Radon). Soit $n=2^{4 \alpha+\beta}(2 \gamma+1)$ avec $0 \leq \beta \leq 3$. Cette décomposition est unique. Soit ensuite $\rho(n)=8 \alpha+2^{\beta}$. Alors il existe $\rho(n)-1$ champs de vecteurs tangents, orthogonaux deux à deux, à la sphère $\mathbb{S}^{n-1}$. Les champs sont donnés explicitement par des matrices orthogonales.

Remarque. Le problème topologique des champs de vecteurs sur les sphères a la même réponse. Il a fallu pour cela attendre 1962 (Frank Adams) pour une démonstration. Mais c'est la démarche d'Eckmann, qui consiste en fait à inverser la fonction $\rho(n)$, qui a lancé la recherche dans la bonne direction.

Eckmann devait présenter au séminaire l'article de Radon, qu'il avait trouvé particulièrement rébarbatif, bien que la solution fournisse explicitement les champs de vecteurs. La démarche de Radon mérite cependant attention (remarquons que le résultat de Radon est purement algébrique, et ne nécessite aucun recours à la topologie).

i) Notons $I$ la matrice-unité $(n \times n)$. S'il existe une matrice orthogonale $A$ ayant la propriété que $x I+y A$ est orthogonale pour tous $(x, y)$ avec $x^{2}+y^{2}=1$, on montre facilement que $A$ est antisymétrique de carré $-I$. Une conséquence immédiate est que $n$ doit être pair. C'est donc la version orthogonale du théorème du hérisson.

ii) On prend $x I+y A+z B$ comme combinaison. Comme précédement, on obtient $A^{2}=B^{2}=A B A^{-1} B^{-1}=-I$. Un raisonnement ad hoc montre alors que la dimension doit être divisible par 4, et que la famille $I, A, B, A B$ est orthogonale (si une sphère a deux champs de vecteurs tangents orthogonaux, elle en a un troisième).

La construction explicite des matrices montre ensuite que les familles orthogonales successives se trouvent soit dans la même dimension, soit dans la dimension double. On reste donc dans les puissances de 2. Mais les distinctions de cas rendent le travail fastidieux.

Mettons-nous dans la situation d'Eckmann. Son sujet de recherche est le problème topologique des champs de vecteurs sur les sphères, le travail de Radon est donc incontournable. Il fait alors la constatation suivante, qui est tout à fait visible sur le cas particulier ii).

Les matrices $A$ et $B$ engendrent un groupe fini $( \pm I, \pm A, \pm B, \pm A B)$ d'ordre 8 (cas général : une puissance de 2), isomorphe au groupe des unités des quaternions $( \pm 1, \pm i, \pm j$, $\pm k$ ). Elles constituent par conséquent une représentation de dimension $n$ du groupe des quaternions. De plus, la matrice $-I$ est un commutateur $\left(A B A^{-1} B^{-1}=-I\right)$.

En 1937, I. Schur, venu d'Allemagne, était venu à Zurich donner un cours de théorie des représentations des groupes. J'ai eu en main le polycopié de ce cours, rédigé par Stiefel. Il me paraît certain qu'Eckmann en disposait en 1940. Le résultat principal de la théorie est le théorème d'orthogonalité des caractères, dont on déduit en particulier :

La représentation régulière (table de multiplication!) d'un groupe fini se décompose, sur les nombres complexes, en autant de représentations irréductibles qu'il y a de classes 
conjuguées dans le groupe, avec chaque représentation irréductible apparaissant autant de fois que sa dimension. Toute représentation irréductible figure dans la représentation régulière. De plus, le nombre de représentations irréductibles de dimension 1 (caractères) est égal à l'ordre de l'abélianisé du groupe (ici 4). A cet argument général s’ajoute, dans notre cas, le fait que la matrice - I est un commutateur. Il n'y a donc aucune représentation de dimension 1 dans la décomposition! C'est le lemme de Schur. Comme l'équation des dimensions est $8=1^{2}+1^{2}+1^{2}+1^{2}+2^{2}$, cela a pour conséquence que la représentation donnée est de dimension 2 sur les nombres complexes. Il reste à voir qu'une représentation de dimension 2 du groupe des quaternions ne peut pas être réelle, ce qui est assez facile. On obtient donc directement le fait que la dimension doit être divisible par 4, retrouvant le résultat de Radon.

Eckmann traite ensuite complètement le cas général, retrouvant tous les résultats de Radon. Son article, paru en 1942 dans les Commentarii, est un document pédagogique exceptionnel. On peut le donner tel quel comme exercice (avancé, il est vrai) dans un cours de théorie des groupes.

Il y a une partie plus difficile : c'est de décider quand une représentation complexe est équivalente à une représentation réelle de même dimension. C'était un ancien résultat de Frobenius, mis à la page par Schur. Il est occulté aujourd'hui, car il est énoncé à l'aide de l'opération d'Adams $\psi^{2}$, mais n'en est pas moins assez délicat.

Remarque. Il est facile de vérifier que $\rho(n)=n \Longleftrightarrow n=1,2,4,8$. Ce résultat, qui donne les sphères orthogonalement parallélisables, avait été obtenu par Hurwitz en 1898 déjà.

Une bonne généralisation des quaternions est donnée par les algèbres de Clifford, un des plus incontournables outils de travail de la topologie. Il se trouve que les groupes envisagés par Eckmann sont en fait les groupes des unités dans les algèbres de Clifford. Dans un livre de J.F. Adams, 25 ans plus tard, un chapitre est consacré à la classification des algèbres de Clifford, et l'auteur conseille au lecteur de prendre directement l'article d'Eckmann pour se familiariser avec le problème. Un bel exemple de pérennité, surtout si l'on songe qu'Adams a résolu la plupart des problèmes de la topologie algébrique des années 40.

\section{Quelques commentaires}

Le contexte unificateur de toutes les questions abordées ici est la fameuse identité

$$
\left(x_{1}^{2}+x_{2}^{2}\right) \cdot\left(y_{1}^{2}+y_{2}^{2}\right)=\left(x_{1} y_{1}-x_{2} y_{2}\right)^{2}+\left(x_{1} y_{2}+x_{2} y_{1}\right)^{2}
$$

obtenue, par exemple, à l'aide de la multiplication des nombres complexes. ${ }^{3}$ L'équation matricielle qui décrit la genèse de cette identité est

$$
\left(x_{1}\left(\begin{array}{ll}
1 & 0 \\
0 & 1
\end{array}\right)+x_{2}\left(\begin{array}{cc}
0 & -1 \\
1 & 0
\end{array}\right)\right)\left(\begin{array}{l}
y_{1} \\
y_{2}
\end{array}\right)=\left(\begin{array}{l}
x_{1} y_{1}-x_{2} y_{2} \\
x_{1} y_{2}+x_{2} y_{1}
\end{array}\right)
$$

3. En $1962=39^{2}+21^{2}$, année de mon diplôme au Poly, chacun de nous connaissait cette décomposition, car nous savions que Hopf, à l'examen d'algèbre, demandait rituellement si l'année courante était une somme de deux carrés. Le lien avec la remarque ci-dessus est $1962=2 \cdot 9 \cdot 109=\left(1^{2}+1^{2}\right) \cdot 3^{2} \cdot\left(10^{2}+3^{2}\right)$. 
Si $\left(x_{1}, x_{2}\right)$ et $\left(y_{1}, y_{2}\right)$ sont des vecteurs-unité, on obtient deux matrices orthogonales dont toute combinaison linéaire est orthogonale, et conserve donc les longueurs, d'où l'identité de départ. On se trouve donc dans le contexte Hurwitz-Radon-Eckmann. D'autre part, le coefficient binomial $\left(\begin{array}{l}2 \\ 1\end{array}\right)=2$ est pair, on se trouve donc aussi dans le contexte du résultat de Stiefel. Comme le vecteur $\left(-x_{2}, x_{1}\right)$ est constamment perpendiculaire au vecteur $\left(x_{1}, x_{2}\right)$, cela signifie finalement que la sphère $\mathbb{S}^{1}$ (cercle!) est parallélisable.

Soit $f: \mathbb{R}^{r} \times \mathbb{R}^{s} \rightarrow \mathbb{R}^{n}$ une application bilinéaire. On notera $[r, s, n]$ si $f$ est nonsingulière $(f(x, y)=0 \Rightarrow x=0$ ou $y=0)$, et $[[r, s, n]]$ si $f$ est normée $(|f(x, y)|=|x|$. $|y|)$. Clairement $[[r, s, n]] \Rightarrow[r, s, n]$. L'identité ci-dessus est $[[2,2,2]]$. Une excellente référence est un cours donné par Daniel Shapiro :

$$
\text { www. math. osu. edu/ shapiro/lec1.pdf }
$$

Les résultats mentionnés ci-dessus peuvent alors s'énoncer ainsi :

$[[n, n, n]] \Rightarrow n=1,2,4,8$. (Hurwitz 1898)

$[r, r, n]$ symétrique $\Rightarrow$ Il existe un plongement de $\mathbb{R P}^{r-1}$ dans $\mathbb{R}^{n-1} \Rightarrow n>r+1$. (Hopf 1940)

$[r, r, n] \Rightarrow$ Il existe une immersion de $\mathbb{R} \mathbb{P}^{r-1}$ dans $\mathbb{R}^{n-1}$. (Ginzburg 1964)

Il existe un plongement de $\mathbb{R P}^{r-1}$ dans $\mathbb{R}^{2 r-\alpha(n-1)}$. (Steer 1970)

$[n, n, n] \Rightarrow n$ est une puissance de 2. (Stiefel 1940)

$[n, n, n] \Rightarrow n=1,2,4,8$. (Milnor-Kervaire 1958)

$[[r, n, n]] \Rightarrow r \leq \rho(n)$. (Hurwitz-Radon 1923, Eckmann 1942)

$[r, n, n] \Rightarrow r \leq \rho(n)$. (Adams 1962)

$[r, s, n] \Rightarrow(x+y)^{n}=0$ dans $\mathbb{F}_{2}[x, y] /\left(x^{r}, y^{s}\right)$. (Stiefel 1940)

Pour terminer, un simple exemple montrera que le sujet est d'une richesse insoupçonnée.

$[16,16,23]$ et 23 est minimal, alors que $[[16,16, n]] \Rightarrow n>28$ et que $[[16,16,32]]$. (Kee Lam 1972 et 1985)

\section{Références}

[1] Eckmann, B. : Gruppentheoretischer Beweis des Satzes von Hurwitz-Radon über die Komposition quadratischer Formen. Comment. Math. Helv. 15 (1942-43), 358-366.

[2] Hopf, H. : Systeme symmetrischer Bilinearformen und euklidische Modelle der projektiven Räume. Vierteljahresschrift der Naturforsch. Gesellschaft in Zürich (Festschrift Rudolf Fueter) 85 (1940), 165-177.

[3] Stiefel, E. : Über Richtungsfelder in den projektiven Räumen und einen Satz aus der reellen Algebra. Comment. Math. Helv. 13 (1940-41), 201-218.

François Sigrist

Université de Neuchâtel

Institut de Mathématiques

Rue Emile Argand 11

2009 Neuchâtel, Suisse

e-mail : Francois.Sigrist@unine.ch 